\title{
Riesgo de recurrencia y de nuevas neoplasias cutáneas malignas en sujetos mexicanos con carcinoma basocelular
}

\author{
Risk of recurrence and new malignant cutaneous neoplasms in Mexican subjects with basal cell \\ carcinoma \\ José F. Ruiz-González', Elizabeth Guevara-Gutiérrez¹, Mercedes Hernández-Torres", \\ Guillermo Solís Ledesma ${ }^{1}$ y Alberto Tlacuilo-Parra ${ }^{*}$ \\ 'Instituto Dermatológico de Jalisco Dr. José Barba Rubio, Secretaría de Salud Jalisco, Zapopan; ${ }^{2}$ Unidad Médica de Alta Especialidad Hospital de \\ Pediatría, Centro Médico Nacional de Occidente del Instituto Mexicano del Seguro Social, Guadalajara. Jalisco, México
}

\begin{abstract}
Resumen
Introducción: El carcinoma basocelular (CBC) es la neoplasia cutánea maligna más común. Objetivo: se investigó el riesgo de recurrencia y de nueva neoplasia cutánea maligna después del tratamiento de CBC. Método: Estudio retrospectivo. Fueron identificados los pacientes con diagnóstico histopatológico de CBC primario, de enero de 2007 a diciembre de 2009, y se revisaron los expedientes para investigar el número de recurrencias, la localización, el tipo de tratamiento y la variante histopatológica, determinando nuevas neoplasias cutáneas malignas. El análisis incluyó estadística descriptiva e inferencial, considerando significativa una $p<0.05$. Resultados: Se incluyeron 397 pacientes, con un seguimiento promedio de $4 \pm 1.5$ años. La recurrencia se presentó en el 4\% y se relacionó con un mayor tiempo de evolución (36 vs. 32 meses; $p=0.04$ ) y haber sido tratado mediante técnicas destructivas (electrofulguración, criocirugía o imiquimod; $31 \mathrm{vs.} \mathrm{4 \% ;} p=0.0004$ ). No hubo relación con la localización ni con la variante histopatológica. El riesgo de desarrollar una nueva neoplasia maligna fue del 25\%, y de ellas el $66 \%$ correspondió a un nuevo CBC y el $30 \%$ a carcinoma espinocelular. Conclusiones: Es importante el seguimiento de los pacientes con CBC para identificar tanto las recurrencias como las nuevas neoplasias malignas, independientemente de la localización y de la variante histopatológica del primario. El tratamiento con técnicas quirúrgicas condiciona una menor recaída que las técnicas destructivas.
\end{abstract}

PALABRAS CLAVE: Carcinoma basocelular. Recurrencia. Cáncer cutáneo.

\begin{abstract}
Introduction: Basal cell carcinoma $(B C C)$ is the most common skin malignant neoplasm. Objective. Investigate the risk of recurrence and of new skin malignant neoplasms, after treatment of BCC. Method: Retrospective study. We examined the files of patients with histopathological diagnosis of primary BCC, between January 2007 and December 2009, and we investigate number of recurrences and their relationship with localization, treatment type, and histopathological variant, and the number of new skin malignant neoplasms. For analysis, we employed descriptive and inferential statistics; $p<0.05$ was considered significant. Results: $A$ total of 397 patients, with an average follow-up of $4 \pm 1.5$ years. Recurrences presented in $4 \%$.
\end{abstract}

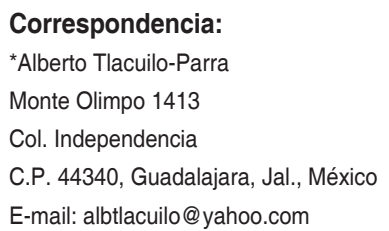

Fecha de recepción: 26-02-2018

Fecha de aceptación: 09-05-2018

DOI: $10.24875 / C I R U .18000190$
Cir Cir. 2018;86:417-422

Contents available at PubMed www.cirugiaycirujanos.com 
Recurrences were related with longer time of evolution (36 vs. 32 months; $p=0.04$ ) and treatment with destructive techniques (electrofulguration, cryosurgery or imiquimod; $31 \mathrm{vs.} \mathrm{4 \% ;} p<0.001$ ). There was no relationship with localization, or the histopathological variant. The risk of developing a new malignant neoplasm was $25 \% ; 66 \%$ corresponded to a new BCC and $30 \%$ to squamous cell carcinoma. Conclusions: Follow-up of patients with BCC should be conducted independently of their localization and histopathological variant, especially in patients with greater evolution time, principally with surgical techniques.

KEY WORDS: Basal cell carcinoma. Recurrence. Skin cancer.

\section{Introducción}

El carcinoma basocelular (CBC) es la neoplasia maligna que más se diagnostica en la práctica dermatológica; corresponde al $65-74 \%$ de todos los tumores cutáneos ${ }^{1-4}$ y su incidencia ha aumentado a lo largo de los años en diferentes países ${ }^{5-7}$. En México se estima una prevalencia de 3.9 por cada 1000 habitantes $^{2,8}$. Aunque tiene un riesgo bajo de producir metástasis, el riesgo de recurrencia varía del 2 al $10 \%^{9-12}$, y el de presentar una nueva neoplasia maligna va del 10.4 al $21.2 \%{ }^{13,14}$. Esto depende de las características clínico-histopatológicas del tumor, siendo de mayor riesgo la localización en la nariz, el párpado, la sien, el hélix o el cuello, el tamaño mayor de $10 \mathrm{~mm}$, el patrón histopatológico morfeiforme 0 un $C B C$ recurrente ${ }^{15}$, y el tipo de tratamiento utilizado, siendo mayor el porcentaje de recurrencia con técnicas destructivas como electrofulguración ${ }^{16}$, criocirugía $(10 \%)^{12}$ o imiquimod $(20.6 \%)^{17}$, mientras que con la extirpación con márgenes predeterminados es menor $(2 \%)^{10,11}$. No encontramos estudios realizados en México a este respecto, por lo que nuestro objetivo fue investigar, en pacientes con diagnóstico de $\mathrm{CBC}$, qué factores se asociaron a recurrencia del $C B C$ y si dicha recurrencia está relacionada con las características clínicas del paciente o del propio tumor, así como determinar la frecuencia de una nueva neoplasia cutánea maligna.

\section{Método}

Estudio retrospectivo en el que se incluyeron pacientes con diagnóstico histopatológico de $\mathrm{CBC}$ primario registrados en la base de datos del Instituto Dermatológico de Jalisco del 1 de enero de 2007 al 31 de diciembre de 2009, que contaran con un seguimiento mínimo de 2 años después del tratamiento del CBC. Se revisaron los expedientes electrónicos para investigar la edad, el sexo y tiempo de evolución, y para determinar el número de recurrencias (evidencia de células tumorales malignas compatibles con CBC sobre la cicatriz del $\mathrm{CBC}$ primario previamente tratado 0 a $2 \mathrm{~cm}$ de ella, y cuando menos 2 meses después del tratamiento $)^{9}$ y de nuevas neoplasias cutáneas malignas (confirmadas histopatológicamente; cuando se trató de un nuevo $\mathrm{CBC}$ que estuviera alejado cuando menos $2 \mathrm{~cm}$ de la cicatriz del $\mathrm{CBC}$ tratado previamente o sobre la cicatriz cuando el diagnóstico fue diferente a $\mathrm{CBC})^{14}$. Se investigó la relación del porcentaje de recurrencias con la localización, el tipo de tratamiento y la variante histopatológica del CBC. Se excluyeron registros de pacientes cuyo diagnóstico histopatológico se obtuvo de una biopsia y no del resultado de la extirpación completa del tumor. Para el análisis de los datos se calcularon el promedio y la desviación estándar. Para la comparación de variables cualitativas se utilizaron la prueba de ji al cuadrado o la prueba exacta de Fisher, según fuera el caso. Para las variables cuantitativas se utilizó la prueba t de Student. Para estimar la relación entre el riesgo de $\mathrm{CBC}$ recurrente con sus características clínicas e histopatológicas se determinó la razón de momios con un intervalo de confianza del $95 \%$. Se consideró significancia estadística una $p<$ 0.05 . Para el procesamiento de los datos se utilizaron los programas Excel de Microsoft 2013 y Epi Info ${ }^{\mathrm{TM}}$ versión 7.0. Esta investigación fue aprobada por el Comité de Ética y de Investigación de la institución.

\section{Resultados}

\section{Características generales}

Durante el periodo de estudio, 397 pacientes con diagnóstico de CBC primario cumplieron los criterios de selección. La edad promedio fue de $65 \pm 13$ años (rango: 16 a 98 años), y 156 (39\%) eran de sexo masculino y $241(61 \%)$ de sexo femenino. El tiempo de evolución promedio del CBC al momento del diagnóstico fue de $33.5 \pm 44$ meses (rango: 1 mes a 20 años). El tiempo de seguimiento promedio fue de $4 \pm 1.5$ años (rango: 2 a 10 años). El tratamiento recibido se agrupó en dos modalidades: quirúrgico (cirugía con márgenes predeterminados o cirugía micrográfica de Mohs), que se utilizó en 378 (95\%) pacientes, y destructivo 


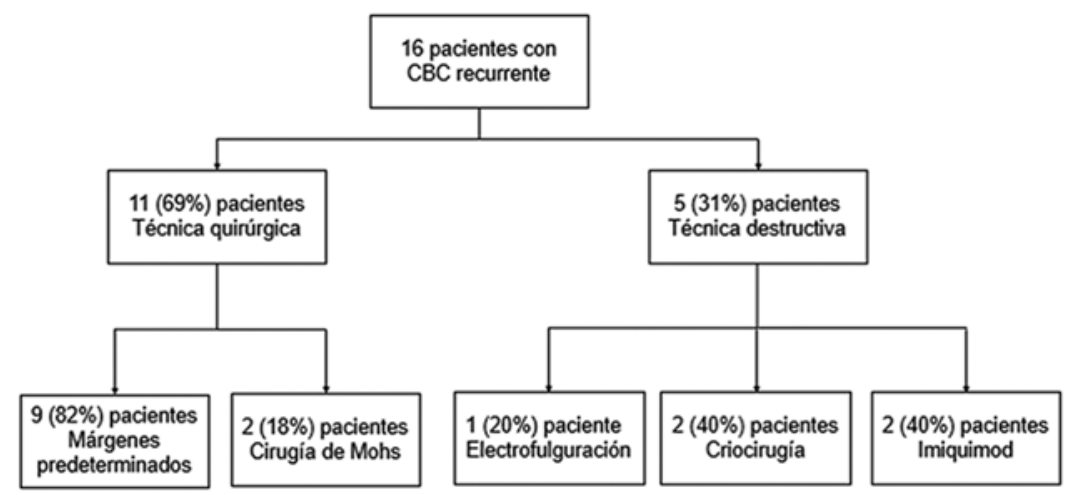

Figura 1. Tratamiento de los carcinomas basocelulares $(C B C)$ recurrentes.

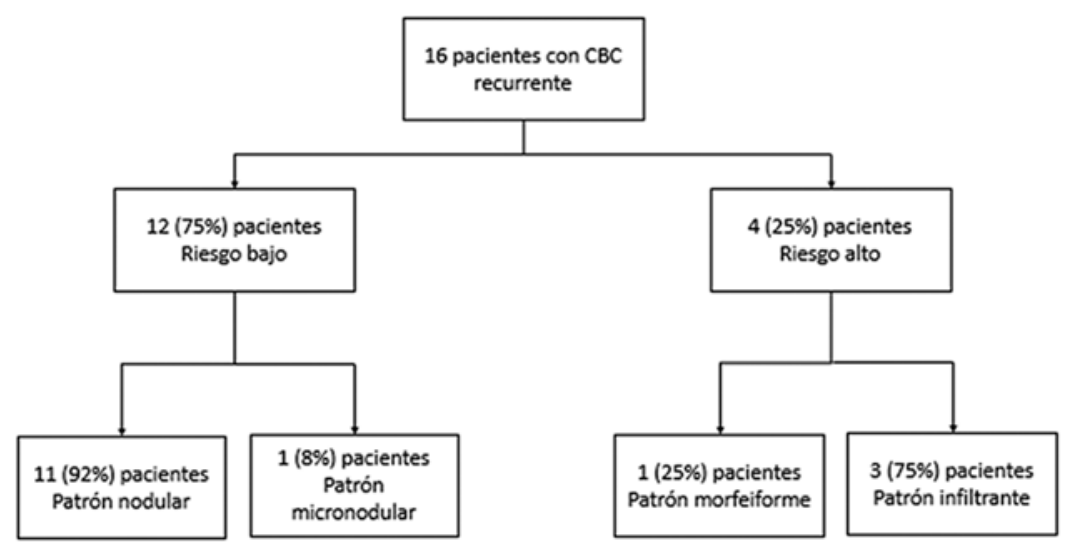

Figura 2. Variantes histopatológicas de los carcinomas basocelulares $(C B C)$ recurrentes.

(electrofulguración, criocirugía e imiquimod), empleado en 19 pacientes $(5 \%)$.

\section{CBC recurrente}

El CBC recurrente se encontró en 16 (4\%) pacientes con una mediana de tiempo de reaparición de 24 meses (rango: 2 meses a 4.8 años). Predominó el sexo femenino, con $10(62.5 \%)$ casos. La mediana de edad fue de 71 años (rango: 47 a 98 años) y la mediana del tiempo de evolución del CBC primario fue de 36 meses (rango: 4 meses a 15 años).

La localización anatómica fue la siguiente: zona $\mathrm{H}$ o de alto riesgo (párpados, cejas, región periorbitaria, nariz, labios en su porción cutánea y bermellón, mentón, región mandibular, áreas preauricular y retroauricular) en $8(50 \%)$ casos, y zona $L$ o de mediano riesgo (piel cabelluda, frente, mejillas y cuello) en $8(50 \%)$ casos. No se observaron recurrencias en la zona $\mathrm{M}$ o de bajo riesgo (tronco y extremidades). En $11(69 \%)$ pacientes se utilizaron técnicas quirúrgicas y en $5(31 \%)$ se utilizaron técnicas destructivas
(Fig. 1). El patrón histopatológico de bajo riesgo estuvo presente en $12(75 \%)$ pacientes, y el de alto riesgo en 4 (25\%) (Fig. 2).

Al comparar el grupo de 16 pacientes con $\mathrm{CBC}$ recurrente y el grupo de 381 pacientes que no tuvieron $C B C$ recurrente, encontramos diferencias estadísticamente significativas en el tiempo de evolución (36 vs. 32 meses; $p=0.04$ ), el uso de técnicas quirúrgicas (69 vs. $96 \% ; p=0.0004)$ y el uso de técnicas destructivas (31 vs. $4 \% ; p=0.0004$ ). En la tabla 1 se presenta la comparación entre variables de los casos con y sin $\mathrm{CBC}$ recurrente.

\section{Nuevas neoplasias cutáneas malignas}

De los 397 pacientes con diagnóstico de CBC primario, 98 presentaron un total de 147 nuevas neoplasias cutáneas malignas (25\%): un nuevo tumor en $70(72 \%)$ pacientes, dos nuevos tumores en 17 (17\%) y tres o más tumores en $11(11 \%)$. El tiempo de aparición promedio fue de $32 \pm 45$ meses (rango: 2 meses a 15 años). De ellos, el CBC se presentó en 97 (66\%) 


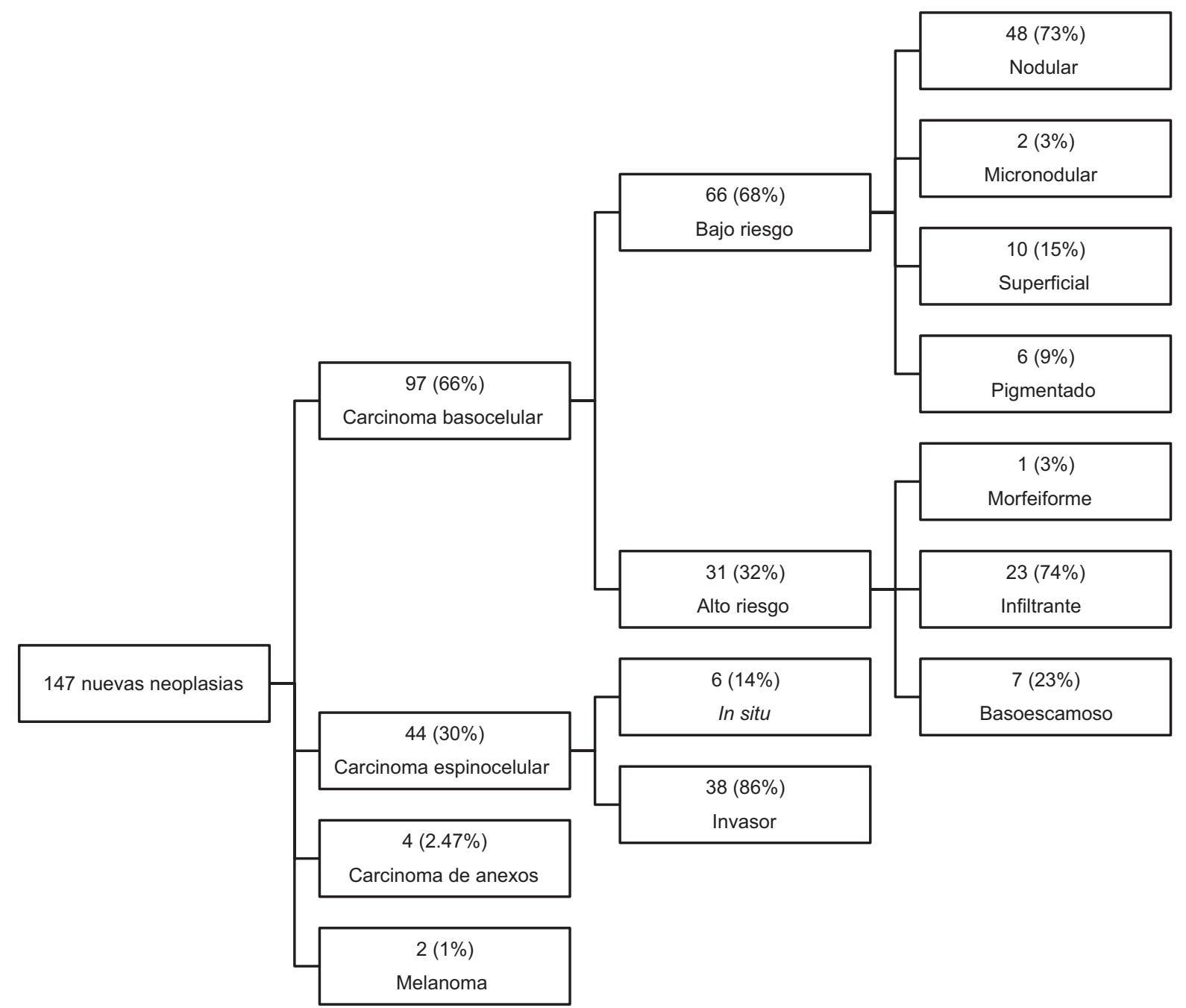

Figura 3. Variantes histopatológicas de las nuevas neoplasias cutáneas malignas.

casos, mientras que se desarrolló un carcinoma espinocelular en $44(30 \%)$. Predominaron los CBC de bajo riesgo, con 66 (68\%) casos, y de ellos el patrón histopatológico más común fue el nodular en 48 (73\%). En la figura 3 se muestran las características histopatológicas de las 147 nuevas neoplasias cutáneas malignas.

Al comparar las características del grupo de pacientes que desarrollaron una nueva neoplasia cutánea maligna con las del grupo de pacientes que no la presentaron, no se hallaron diferencias estadísticamente significativas (Tabla 2).

\section{Discusión}

Este estudio incluyó 397 pacientes con CBC primario, una muestra mayor que la de Marghoob, et al. ${ }^{14}$ de 260 pacientes $^{4}$ y la de McLoone, et al..$^{13}$ de 114 pacientes. La edad promedio fue de 65 años, similar a los 62 años mencionada en otros estudios mexicanos $^{2,8}$. Predominó el sexo femenino, con el $61 \%$ de los casos, lo cual difiere de lo encontrado por otros autores que reportan un predominio del sexo masculino ${ }^{2,8,18}$. El tiempo de evolución del CBC al momento del diagnóstico fue, en promedio, de 33.5 meses, mayor que el reportado por otros autores que mencionan que en la mayoría de los casos el diagnóstico se realiza antes de los 12 meses de evolución ${ }^{19}$. El tiempo de seguimiento posterior al diagnóstico fue, en promedio, de 4 años, menor que el reportado por Chren, et al. ${ }^{9}$, que en promedio fue de 7.4 años, pero mayor que el referido por McLoone, et al. ${ }^{13}$ quienes realizaron un seguimiento de 2 años, algunos de ellos a través de llamadas telefónicas y no de una exploración física. 
Tabla 1. Comparación entre los carcinomas basocelulares recurrentes y no recurrentes

\begin{tabular}{|c|c|c|c|c|}
\hline Variable & $\begin{array}{c}\text { CBC } \\
\text { recurrente } \\
(n=16)\end{array}$ & $\begin{array}{l}\mathrm{CBC} \text { no } \\
\text { recurrente } \\
(\mathrm{n}=381)\end{array}$ & $\mathrm{p}$ & $\begin{array}{c}\text { OR } \\
\text { (IC 95\%) }\end{array}$ \\
\hline Edad en años ( \pm DE) & $71^{*}$ & $65 \pm 12$ & $0.54^{\dagger}$ & - \\
\hline Sexo femenino, $n(\%)$ & $10(62.5)$ & $231(61)$ & $0.88^{\ddagger}$ & $1.08(0.35-3.43)$ \\
\hline Evolución en meses ( $\pm \mathrm{DE})$ & $36^{*}$ & $32 \pm 43$ & $0.04^{+}$ & - \\
\hline Zona alto riesgo, $n(\%)$ & $8(50)$ & $222(58)$ & $0.43^{\ddagger}$ & $0.72(0.24-2.15)$ \\
\hline Zona mediano riesgo, $\mathrm{n}(\%)$ & $8(50)$ & $123(32)$ & $0.13^{\ddagger}$ & $2.10(0.70-6.31)$ \\
\hline Zona bajo riesgo, n (\%) & $0(0)$ & $36(9)$ & $0.38^{\S}$ & - \\
\hline Técnica quirúrgica, n (\%) & $11(69)$ & $367(96)$ & $0.0004^{\S}$ & - \\
\hline Márgenes predeterminados, n (\%) & $9(82)$ & $355(97)$ & $0.05^{\S}$ & - \\
\hline Cirugía de Mohs, n (\%) & $2(18)$ & $12(3)$ & $0.05^{\S}$ & - \\
\hline Técnica destructiva, n (\%) & $5(31)$ & $14(4)$ & $0.0004^{\S}$ & - \\
\hline Patrón histopatológico alto riesgo, $\mathrm{n}$ (\%) & $4(25)$ & $80(21)$ & $0.75^{\S}$ & - \\
\hline Patrón histopatológico bajo riesgo, n (\%) & $12(75)$ & $301(79)$ & $0.75^{\S}$ & - \\
\hline
\end{tabular}

Tabla 2. Comparación entre los grupos con y sin nuevas neoplasias cutáneas malignas

\begin{tabular}{lccc}
\hline Variante & $\begin{array}{c}\text { CBC con } \\
\text { nueva } \\
\text { neoplasia } \\
(\mathbf{n}=\mathbf{9 8})\end{array}$ & $\begin{array}{c}\text { CBC sin } \\
\text { nueva } \\
\text { neoplasia } \\
(\mathbf{n}=\mathbf{2 9 9})\end{array}$ & $\mathbf{p}$ \\
\hline Sexo femenino, $\mathrm{n}(\%)$ & $53(54)$ & $188(63)$ & $0.12^{*}$ \\
Edad, años $( \pm \mathrm{DE})$ & $70 \pm 11$ & $64 \pm 13$ & $2.9^{\dagger}$ \\
Evolución, meses $( \pm \mathrm{DE})$ & $32 \pm 41$ & $34 \pm 45$ & $0.79^{\dagger}$ \\
Seguimiento, meses $( \pm \mathrm{DE})$ & $50 \pm 19$ & $49 \pm 17$ & $0.47^{\dagger}$ \\
$\begin{array}{l}\text { *Ji al cuadrado. } \\
\text { †Prueba t de Student. } \\
\text { CBC: carcinoma basocelular; DE: desviación estándar. }\end{array}$ & & \\
\end{tabular}

El riesgo de $\mathrm{CBC}$ recurrente fue del $4 \%$, semejante a la prevalencia del 3.3\% reportada en población de los EE.UU. en el estudio de Chren, et al. ${ }^{9}$ ya mencionado. En nuestro estudio, la recurrencia se presentó en promedio a los 25 meses del diagnóstico del CBC primario, pero hubo recurrencias hasta los 4.8 años, un tiempo mayor que el reportado por Chren, et al. ${ }^{9}$, que fue de 3.9 años. Este hallazgo es importante, pues en México, de acuerdo con la Guía de práctica clínica para la prevención, diagnóstico y tratamiento del carcinoma basocelular20, la recomiendación es un seguimiento de 3 años en los pacientes de alto riesgo, por lo que quizá sea deseable un seguimiento mayor.
Encontramos un tiempo de evolución significativamente mayor en los pacientes con recurrencia, lo que sugiere que debemos tener especial cuidado ante pacientes con lesiones de larga evolución.

El $50 \%$ de los CBC primarios que recurrieron se encontraban en zonas de alto riesgo, mientras otros autores reportan esta localización en el $81 \%$ de los casos $^{21}$. No encontramos recurrencias en los tumores localizados en zonas de bajo riesgo, lo cual apoya en parte la recomendación de la British Association of Dermatologist de dar seguimiento solo a los pacientes con tumores en sitios anatómicos de alto riesgo o con tipo histológico agresivo ${ }^{22}$.

El riesgo de recurrencia fue significativamente mayor con el uso de técnicas destructivas que con el empleo de técnicas quirúrgicas, lo que corrobora el hecho de que estas tienen un mayor porcentaje de falla del tratamiento ${ }^{23}$. El riesgo de recurrencia en los pacientes tratados con márgenes predeterminados de nuestra población fue del $2.5 \%$, menor que el publicado por Mosterd, et al. ${ }^{21}$ en pacientes holandeses, del $4.1 \%$.

En cuanto a la variante histopatológica de los CBC que recurrieron, en la literatura se menciona que la de alto riesgo es un factor para la presencia de recurrencias $^{15}$, pero nosotros no encontramos diferencia significativa con las de bajo riesgo. Nuestro resultado 
es semejante al publicado por Chren, et al..$^{9}$, quienes reportan que los $\mathrm{CBC}$ con variante histopatológica de bajo riesgo también recurrieron más que aquellos de alto riesgo (3.8 vs. $1.6 \%)$.

Con respecto al riesgo de nuevas neoplasias malignas, en nuestro estudio fue del $25 \%$, mayor que el reportado por McLoone, et al..$^{13}$ en población irlandesa, que fue del $17.5 \%$. El tiempo promedio en que aparecieron las nuevas neoplasias fue de 32 meses, parecido a lo publicado previamente de 38.3 meses $^{14}$. También hubo pacientes que presentaron nuevas neoplasias hasta 15 años después del diagnóstico del CBC primario, por lo que habría que considerar un mayor tiempo de seguimiento y no solo 3 años como se mencionó previamente ${ }^{20}$.

En cuanto al número de nuevas neoplasias por paciente, solo el $11 \%$ tuvo tres o más. Por el contrario, Marghoob, et al. ${ }^{14}$, en 260 pacientes de los EE.UU., reportaron que el $21.2 \%$ presentaron tres o más tumores. Esta mayor frecuencia puede deberse a que solo incluyeron pacientes de piel blanca, la cual tiene una mayor predisposición para el desarrollo de cáncer ${ }^{24}$.

La nueva neoplasia cutánea maligna más frecuente fue el CBC, en el $66 \%$ de los casos, seguida del carcinoma espinocelular en el $30 \%$. Ambos tumores también son mencionados por McLoone, et al. ${ }^{13}$, aunque con frecuencias menores del $16.7 \%$ y del $2.6 \%$, respectivamente. Es importante mencionar que en este último estudio, aunque el tiempo de seguimiento fue de 2 años, al $47 \%$ se les contactó telefónicamente y no fueron valorados por ningún médico, lo que podría explicar estas diferencias.

En los nuevos CBC predominó el patrón histopatológico de bajo riesgo, en el $68 \%$ de los casos, dato no especificado en las series previamente publicadas. Al analizar las características epidemiológicas de los pacientes con una nueva neoplasia cutánea maligna, tales como sexo, edad y tiempo de evolución o de seguimiento, no encontramos diferencias significativas al compararlas con las de aquellos que no la presentaron.

\section{Conflicto de intereses}

$$
\text { Ninguno por declarar. }
$$

\section{Bibliografía}

1. Lucas R, McMichael T, Smith W, Armstrong B. A systematic review of worldwide incidence of nonmelanoma skin cancer. $\mathrm{Br} \mathrm{J}$ Dermatol. 2012;166:1069-80.

2. Hernández-Zárate SI, Medina-Bojórquez A, López-Tello SAL, Alcalá-Pérez D. Epidemiología del cáncer de piel en pacientes de la Clínica de Dermato-oncología del Centro Dermatológico Dr. Ladislao de la Pascua. Estudio retrospectivo de los últimos ocho años. Dermatol Rev Mex. 2012;56:30-7.

3. Gutiérrez VRM. Cáncer de piel. Rev Fac Med UNAM. 2003:46:166-71.

4. Alfaro A, Castrejón L, Rodríguez OM. Cáncer de piel. Estudio epidemiológico a 10 años en derechohabientes del ISSSTE en Nuevo León. Dermatol Rev Mex. 2010;54:321-5.

5. Holme S. Changing trends in non-melanoma skin cancer in South Wales, 1988-1998. Br J Dermatol. 2000;143:1224-9.

6. Demers AA, Nugent Z, Mihalcioiu C, Wiseman MC, Kliewer EV. Trends of nonmelanoma skin cancer from 1960 through 2000 in a Canadian population. J Am Acad Dermatol. 2005;53:320-8.

7. Green A, Battistutta D, Hart V, Leslie D, Weedon D. Skin cancer in a subtropical Australian population: incidence and lack of association with occupation. Am J Epidemiol. 1996:144:1034-40.

8. Saucedo RAP, Gutiérrez VRM, Medina BA. Cáncer de piel en el Centro Dermatológico Pascua. Estudio epidemiológico de 10 años. Dermatol Rev Mex. 2005;49:231-40.

9. Chren MM, Linos E, Torres JS, Stuart SE, Parvataneni R, Boscardin WJ Tumor recurrence 5 years after treatment of cutaneous basal cell carcinoma and squamous cell carcinoma. J Invest Dermatol. 2013;133:1188-96.

10. Walker P, Hill D. Surgical treatment of basal cell carcinomas using standard postoperative histological assessment. Australas J Dermatol. 2006; $47: 1-12$.

11. Griffiths RW, Suvarna SK, Stone J. Do basal cell carcinomas recur after complete conventional surgical excision? $\mathrm{Br} J$ Plast Surg. 2005;58:795-805.

12. Kuflik EG. Evidence-based review of the use of cryosurgery in treatment of basal cell carcinoma. Dermatol Surg. 2003;29:566-71.

13. McLoone NM, Tolland J, Walsh M, Dolan OM. Follow-up of basal cell carcinomas: an audit of current practice. J Eur Acad Dermatol Venereol. 2006;20:698-701.

14. Marghoob A, Kopf AW, Bart RS, Sanfilippo L, Silverman MK, Lee P, et al. Risk of another basal cell carcinoma developing after treatment of a basal cell carcinoma. J Am Acad Dermatol. 1993;28:22-8.

15. Batra RS, Kelley LC. A risk scale for predicting extensive subclinical spread of nonmelanoma skin cancer. Dermatol Surg. 2002;28:107-12.

16. Rodríguez-Vigil T, Vázquez-López F, Pérez-Oliva N. Recurrence rates of primary basal cell carcinoma in facial risk areas treated with curettage and electrodesiccation. J Am Acad Dermatol. 2007;56:91-5.

17. Schulze HJ, Cribier B, Requena L, Reifenberger J, Ferrándiz C, García Díez $\mathrm{A}$, et al. Imiquimod $5 \%$ cream for the treatment of superficial basal cell carcinoma: results from a randomized vehicle-controlled phase III study in Europe. Br J Dermatol. 2005;152:939-47.

18. Roewert-Huber J, Lange-Asschenfeldt B, Stockfleth E, Kerl H. Epidemiology and aetiology of basal cell carcinoma. $\mathrm{Br} J$ Dermatol. 2007;157:S47-S51.

19. Kricker A, Armstrong B, Hansen V, Watson A, Singh-Khaira G, Lecathelinais $C$, et al. Basal cell carcinoma and squamous cell carcinoma growth rates and determinants of size in community patients. J Am Acad Dermatol. 2014;70:456-64.

20. Guía de Práctica Clínica de Prevención, Diagnóstico y Tratamiento del Carcinoma Basocelular. México: Secretaría de Salud; 2013.

21. Mosterd K, Krekels GA, Nieman FH, Ostertag JU, Essers BA, Dirksen $C D$, et al. Surgical excision versus Mohs' micrographic surgery for primary and recurrent basal-cell carcinoma of the face: a prospective randomised controlled trial with 5-years' follow-up. Lancet Oncol. 2008;9:1149-56.

22. Telfer NR, Colver GB, Morton CA, British Association of Dermatologists. Guidelines for the management of basal cell carcinoma. Br J Dermatol. 2008;159:35-48.

23. Ceilley RI, Del Rosso JQ. Current modalities and new advances in the treatment of basal cell carcinoma. Int J Dermatol. 2006;45:489-498.

24. Chinem VP, Miot HA. Prevalence of actinic skin lesions in patients with basal cell carcinoma of the head: a case-control study. Rev Assoc Med Bras. 2011;58:188-96. 